\title{
Activación de Recursos en Psicoterapia. ¿Cómo se ha estudiado y qué sabemos?: Una revisión sistemática
}

\section{Resource Activation in Psychotherapy. How has it been studied and what do we know?: A Systematic Review}

\author{
José Domingo Salvo Rivera ${ }^{1} \underline{\mathrm{ORCID}}$
}

Pontificia Universidad Católica de Chile

Instituto Milenio para la Investigación en Depresión y Personalidad

Chile

Fecha correspondencia:

Recibido: noviembre 16 de 2019.

Aceptado: septiembre 29 de 2020.

Forma de citar:

Salvo Rivera, J.D. (2020). Activación

de Recursos en Psicoterapia.

¿Cómo se ha estudiado y

qué sabemos?: Una revisión

sistemática. Rev. CES Psico, 13(3),

33-50.

Open access

(c) Copyright

Licencia creative commons

Etica de publicaciones

Revisión por pares

Gestión por Open Journal System

DOl: http://dx.doi.org/10.21615/

cesp.13.3.3

ISSN: 2011-3080

Sobre los autores:

1. Magister en Psicoterapia.

Psicólogo. Supervisor clínico en

el Centro de Entrenamiento en

Psicoterapia y Coaching -MIP-

Santiago de Chile.

\section{Resumen}

A partir de los primeros metaanálisis sobre efectividad en psicoterapia, Grawe (1997) abstrae teóricamente cuatro mecanismos transteóricos relacionados con el cambio, siendo el principal de ellos Activación de Recursos. Este mecanismo es entendido como el uso de las capacidades y disposición motivacional del paciente al servicio de un objetivo terapéutico, así como el ajuste de las intervenciones terapéuticas a dichos aspectos. Basado en una revisión sistemática de la literatura en inglés y español, el presente artículo discute resultados de distintos estudios empíricos, con el objetivo de esclarecer qué sabemos sobre la relación entre Activación de Recursos y el cambio en psicoterapia. La búsqueda de artículos no se restringió por año de publicación ni diseño metodológico, en tanto en el estudio reportado se hubiera evaluado el mecanismo Activación de Recursos y se aportara evidencia de su relación con una segunda variable. Los 14 estudios incluidos ofrecen evidencia mayoritariamente a favor de la relación entre Activación de Recursos y resultados terapéuticos, pero con dudas respecto de cómo y cuándo este mecanismo favorece, o incluso pudiera entorpecer el cambio. Estas dudas son discutidas a partir de la relación entre Activación de Recursos y distintas variables de proceso, así como de aspectos metodológicos de los estudios. La principal limitación de esta revisión guarda relación con la heterogeneidad metodológica de los estudios incluidos, sumado a la homogeneidad geográfico-cultural donde fueron desarrollados, lo que sugiere interpretar con cautela tanto la integración de la evidencia como su generalización, a la espera de nuevos estudios en distintos contextos.

Palabras clave: Activación de Recursos, Cambio Terapéutico, Mecanismos Genéricos de Cambio, Psicoterapia, Revisión Sistemática.

\section{Abstract}

From the first meta-analyzes on effectiveness in psychotherapy, Grawe (1997) theoretically abstracts four trans-theoretical mechanisms related to change, the main one being Resource Activation. This is understood as the use of the patient skills and motivational readiness in the service of a therapeutic objective, as well as the adjustment of therapeutic interventions to 
El interés teórico y clínico respecto del uso terapéutico de los recursos y aspectos sanos de los consultantes tiene una larga trayectoria en psicoterapia y otras disciplinas terapéuticas como el counselling psicológico, coaching, trabajo social, etc. (Gelso \& Woodhouse, 2003). these aspects. Based on a systematic review of English and Spanish literature, this article discusses the results of different empirical studies, with the aim of clarifying what we know about the relationship between resource activation and change in psychotherapy. For this review, the search was not restricted by year of publication or methodological design, as long as the Resource Activation mechanism was evaluated and evidence of its relationship with a second variable was provided. The 14 included studies offer evidence mostly in favor of the relationship between Resource Activation and therapeutic results, but with doubts about how and when this mechanism favors or could even hinder change. These doubts are discussed based on the relationship between Resource Activation and different process variables, as well as methodological aspects of the studies. The main limitation of this review is related to the methodological heterogeneity of the included studies, added to the geographical-cultural homogeneity where they were developed, which suggests interpreting with caution both the integration of the evidence and its generalization, pending new studies in different contexts.

Keywords: Resource Activation, Generic Mechanisms of Change, Psychotherapy, Therapeutic Change, Systematic Review.

\section{Introducción}

Desde mediados del siglo pasado hasta la fecha se ha acumulado evidencia suficiente sobre la efectividad de la psicoterapia para contribuir a mejorar la calidad de vida de los consultantes, así como, la inexistencia de diferencias de efectividad entre distintos enfoques terapéuticos (Eysenck, 1994; Lambert \& Ogles, 2004; Smith \& Glass, 1977; Wampold \& Imel, 2015). A partir de esto, durante de los años 80's del siglo XX, y en adelante, el interés comenzó a centrarse en estudios de procesos y proceso-resultado en psicoterapia, dando lugar a distintas propuestas conceptuales y metodológicas cuyo foco esencial es esclarecer los cambios que tienen lugar al interior de la terapia, y responder a las preguntas: ¿qué es lo que cambia? y ¿cómo cambia? Con un fuerte énfasis en el estudio de factores y mecanismos comunes a distintos enfoques terapéuticos (Krause \& Altimir, 2016).

\section{Activación de recursos en psicoterapia}

El interés teórico y clínico respecto del uso terapéutico de los recursos y aspectos sanos de los consultantes tiene una larga trayectoria en psicoterapia y otras disciplinas terapéuticas como el counselling psicológico, coaching, trabajo social, etc. (Gelso \& Woodhouse, 2003). Desde principios del presente siglo, se ha observado un auge en la literatura académica sobre este tema, potenciado por al menos tres líneas de investigación, a saber: 1) El interés por el estudio de mecanismos genéricos o transteóricos, relacionados con el cambio en psicoterapia (Scheel, Davis, \& Henderson, 2012); 2) El interés por sistematizar empíricamente las prácticas de terapias que se agrupan bajo los nombres de "Aproximación de las Fortalezas", "Terapias Centradas en las Fortalezas" o "Terapias Postmodernas" (Gelso \& Woodhouse, 2003; Tarragona, 2006; 2013), y 3) El auge de la Psicología Positiva (Seligman \& Csikszentmihalyi, 2000; Lopez, 2008). En el marco de la primera línea de investigación mencionada, Grawe (Grawe, Donati \& Bernauer,1994; Grawe, 1997), basado en los primeros meta-analisis sobre efectividad en psicoterapia, abstrae teóricamente cuatro mecanismos genéricos relacionados con el cambio terapéutico, a saber: Maestría; Clarificación; Activación de problemas; y Activación de Recursos. Este último mecanismo, sobre el que centramos la atención en el presente artículo, es definido como el uso intencionado de las capacidades y disposiciones motivacionales del paciente al servicio de un objetivo terapéutico, así como 
el ajuste de las intervenciones del terapeuta a dichos aspectos (Grawe, 1997). Algunos ejemplos de intervenciones típicas de este mecanismo incluyen métodos específicos centrados en los recursos de los pacientes provenientes de la Terapia Sistémica y Terapia Centrada en las Soluciones (ej: preguntas de excepciones, reencuadre de problemas, exploración de soluciones, etc.), así como, en términos más generales, la adaptación de las técnicas y métodos del tratamiento a las experiencias particulares de los pacientes (Flückiger, Caspar, Grosse Holtforth, \& Willutzki, 2009).

A partir de la conceptualización de Grawe (1997) del mecanismo Activación de Recursos y el resto de los mecanismos genéricos relacionados con el cambio, se han desarrollado diversos estudios orientados a dilucidar la relación entre dichos mecanismos y los resultados en psicoterapia, sin embargo, hasta la fecha, no existen revisiones que sinteticen y discutan los resultados de estos estudios, permitiendo contribuir a una visión panorámica de cómo este mecanismo puede ser usado por los terapeutas al servicio del cambio (Castronovo \& Hirsch, 2020; Gassmann \& Grawe, 2006; Gelso \& Woodhouse, 2003; Harbin, Gelso, \& Rojas, 2014; Hirsch, Castronovo, \& Santangelo, 2016). El presente trabajo se propone responder a las preguntas: ¿Cómo se ha estudiado el mecanismo Activación de Recursos? y ¿Qué sabemos sobre su relación con otras variables?

\section{Metodología}

La presente revisión fue desarrollada siguiendo las directrices de la metodología PRISMA (Moher, Liberati, Tetzlaff, Altman, \& The PRISMA Group, 2009) para la publicación de revisiones sistemáticas.

\section{Criterios de búsqueda}

La búsqueda de artículos se realizó en las siguientes bases de datos: PsycINFO (EBSCO), PsycARTICLES (EBSCO) y Psychology Database (PROQUEST). Para todas ellas se utilizaron los siguientes términos de búsqueda en inglés: Resource AND Psychotherapy OR Counselling AND Measure; y en español: Recursos Y Psicoterapia 0 Counselling $O$ Consejería Y Medición. Posteriormente se realizó una búsqueda por los autores que participaran en más de un artículo identificado (Smith, Grawe, Flckiger, Grosse Holtforth, Schrammm, Znoj, Mander, Wittorf, Schlarb, Hautzinger, Zipfel, Sammet, y Teufel). Todas las búsquedas se realizaron para todos los campos de los artículos. Sólo se incluyeron artículos publicados en revistas indexadas, pero no se restringió por cuartil. Doce de los catorce artículos finalmente incluidos en la revisión sistemática fueron publicados en revistas de primer cuartil, uno en segundo cuartil y uno en cuarto cuartil. No se restringió tampoco por fecha de publicación ni metodología de estudio, en tanto se hubiera utilizado una operacionalización explícita de la variable Activación de Recursos, congruente con la definición de Grawe (1997).

\section{Criterios de inclusión y exclusión}

Para realizar la selección final de los artículos se consideraron los siguientes criterios de inclusión: (a) El estudio utilizó algún instrumento u operacionalización que permita evaluar el grado en que el trabajo terapéutico se enfoca en el mecanismo de Activación de Recursos; (b) La operacionalización de la variable Activación de Recursos es congruente con la definición de Grawe (1997) (c) El diseño del estudio permite distinguir algún tipo de efecto o relación de Activación de Recursos con al menos una variable adicional. 
Por otro lado, se consideró como criterio de exclusión que la publicación fuese una revisión bibliográfica o teórica del tema y que estuviera en un idioma distinto al inglés o español.

A partir de la búsqueda realizada se identificó un total inicial de 111 artículos. Luego de eliminar los resultados duplicados, y aplicar los criterios de inclusión y exclusión, se seleccionaron 14 artículos. El proceso de búsqueda y selección de los artículos se resumen en la Figura 1.

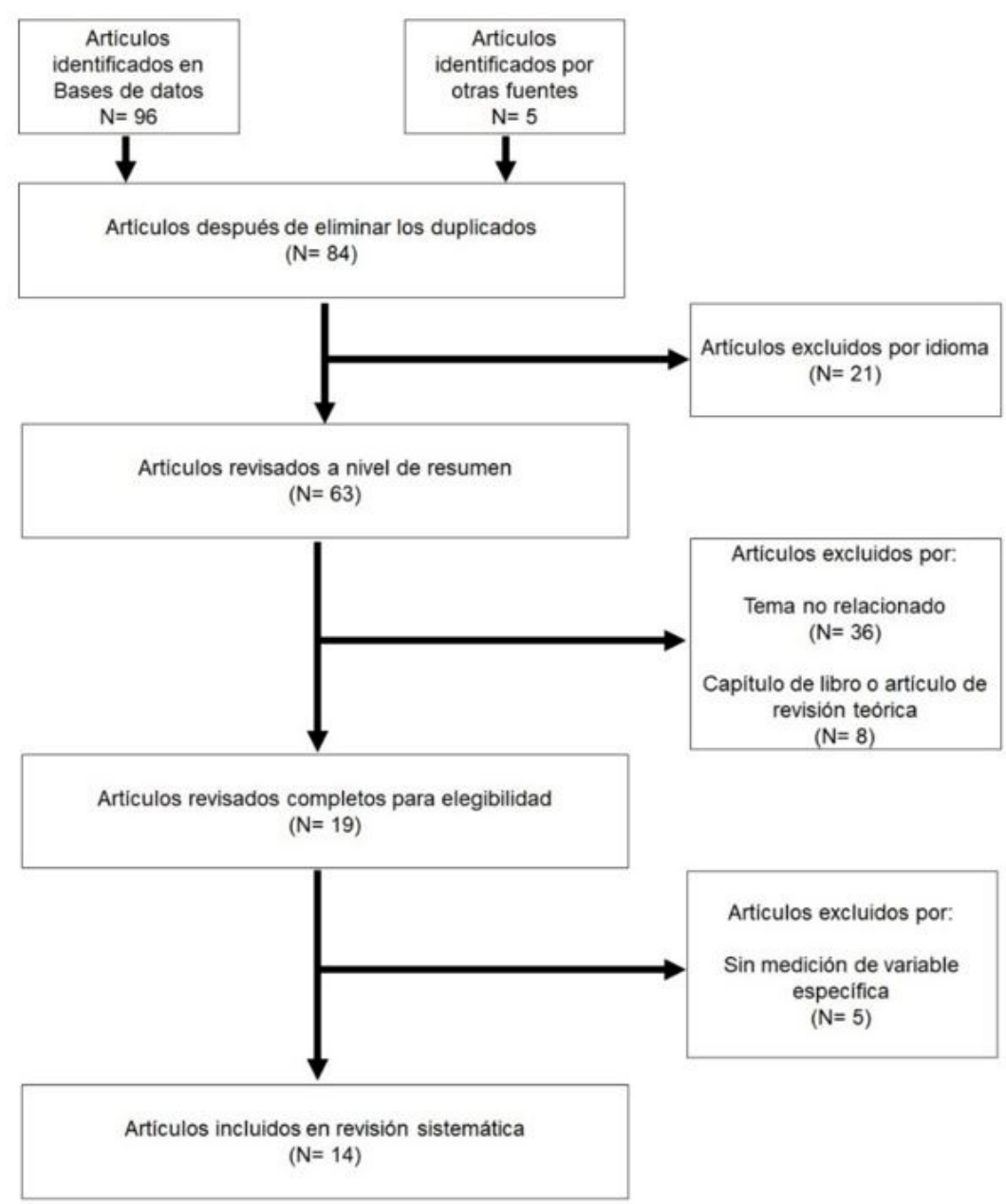

Figura 1. Flujograma del proceso de selección del estudio

\section{Codificación de los estudios}

Se desarrolló una planilla de extracción de datos basada en la propuesta de Lipsey y Wilson (2001), la cual se probó con cuatro artículos, y luego se adaptó la versión final. Los artículos que cumplieron con los criterios de inclusión y exclusión fueron codificados por el autor del presente artículo usando dicha planilla. A partir de este proceso se extrajeron las siguientes características, las cuales se resumen en la Tabla 1, a saber: a) Año; b) Autor; c) País; d) Instrumento utilizado para evaluar la variable específica Activación de Recursos; e) Otras variables que aportan evidencia de su relación con Activación de Recursos, y; f) Tamaño muestral. 
Tabla 1. Artículos incluidos en la revisión sistemática

\begin{tabular}{|c|c|c|c|c|c|}
\hline Año & Autores & País & Instrumento AR & Otras variables & $N$ \\
\hline 2003 & Smith \& Grawe & Suiza & TSA & MGG; AT & 128 \\
\hline 2005 & Smith \& Grawe & Suiza & TSA & MGG; AT & 133 \\
\hline 2006 & Gassmann \& Grawe & Suiza & CMP & $\begin{array}{l}\text { Resultados } \\
\text { terapéuticos finales }\end{array}$ & 30 \\
\hline 2008 & $\begin{array}{c}\text { Flückiger \& Grosse } \\
\text { Holtforth }\end{array}$ & Suiza & $\begin{array}{l}\text { BPSR-T } \\
\text { CMP }\end{array}$ & $\begin{array}{l}\text { Resultados terapéuticos } \\
\text { finales; MGG; AT }\end{array}$ & 40 \\
\hline 2009 & $\begin{array}{l}\text { Flückiger, Caspar, } \\
\text { Holtforth \& Willutzki }\end{array}$ & Alemania & ROMA-T/P & $\begin{array}{c}\text { Resultados terapéuticos } \\
\text { tempranos; } \\
\text { MGG; AT }\end{array}$ & 36 \\
\hline 2010 & $\begin{array}{c}\text { Stangier, von } \\
\text { Consbruc, Schramm } \\
\text { \& Heidenreich }\end{array}$ & Alemania & BPSR-T/ P & $\begin{array}{l}\text { Resultados } \\
\text { terapéuticos finales }\end{array}$ & 62 \\
\hline 2010 & Znoj et al. & Suiza & FEPIG & $\begin{array}{c}\text { Resultados } \\
\text { terapéuticos en fases } \\
\text { temprana, media y final }\end{array}$ & 81 \\
\hline 2013 & Mander et al. & Alemania & SACiP & $\begin{array}{c}\text { Resultados } \\
\text { terapéuticos en fases } \\
\text { temprana, media y final }\end{array}$ & 296 \\
\hline 2013 & $\begin{array}{c}\text { Sander, Zobel, } \\
\text { Dykierek \& Schramm }\end{array}$ & Alemania & BPSR-T/P & $\begin{array}{l}\text { Resultados } \\
\text { terapéuticos finales }\end{array}$ & 30 \\
\hline 2014 & $\begin{array}{l}\text { Flückiger, Zinbarg, } \\
\text { Znoj \& Ackert }\end{array}$ & Suiza & ROMA-P & $\begin{array}{l}\text { Resultados terapéuticos } \\
\text { finales; MGG }\end{array}$ & 20 \\
\hline 2014 & Mander et al. & Alemania & SACiP & $\begin{array}{l}\text { Resultados terapéuticos } \\
\text { finales; EC }\end{array}$ & 296 \\
\hline 2015 & Mander et al. & Alemania & SACiP & $\begin{array}{c}\text { Resultados terapéuticos } \\
\text { finales; EC; EMT }\end{array}$ & 112 \\
\hline 2015 & Mander et al. & Alemania & ITPQ & $\begin{array}{l}\text { Resultados } \\
\text { terapéuticos finales }\end{array}$ & 504 \\
\hline 2018 & Schaller et al. & Alemania & FEPIG & $\begin{array}{l}\text { Resultados terapéuticos } \\
\text { finales; EC }\end{array}$ & 140 \\
\hline
\end{tabular}

Nota: País= País de realización del estudio; Instrumento AR= Instrumento utilizado para evaluar el mecanismo Activación de Recursos: N= Tamaño muestral; MGG= Mecanismos genéricos descritos por Grawe (1997); AT = Alianza Terapéutica (Bordin, 1976); EMT= Esquemas Maladaptativos Tempranos (Young, 1990); EC= Etapas de Cambio (Prochaska \& DiClemente, 1983)

\section{Resultados}

\section{Características de los artículos incluidos}

El rango temporal de publicación de los catorce artículos incluidos en la presente revisión abarca quince años, desde 2003 hasta 2018. Dos autores, Klaus Grawe y Johannes Mander, en conjunto, participan en nueve artículos. Ocho de los artículos incluidos reportan estudios desarrollados en Alemania y seis en Suiza. Respecto de la metodología utilizada, cuatro artículos corresponden a diseños de estudio aleatorizados, mientras que los otros diez muestran alta heterogeneidad de diseños.

Respecto de las muestras de los artículos incluidos, se observa un tamaño muestral con un rango que va desde 20 hasta 504 participantes. Todos los pacientes incluidos son germanoparlantes, mayores de edad. Diez de los catorce artículos reportan datos de pacientes ambulatorios y cuatro en tratamiento residencial hospitalario. 
En términos de diagnóstico, se incluyen pacientes con una alta heterogeneidad en cuanto a trastornos psicopatológicos y grados de severidad, incluyendo trastornos del ánimo, de ansiedad y psicosomáticos. En ningún estudio se incluyen pacientes con trastornos psicóticos ni con trastornos relacionados con sustancias.

\section{Instrumentos para evaluar Activación de Recursos}

En los estudios reportados en los catorce artículos incluidos se observó el uso de siete instrumentos distintos para evaluar el mecanismo de Activación de Recursos, los cuales son resumidos en la Tabla 2.

Tabla 2. Instrumentos utilizados en artículos incluidos

\begin{tabular}{cccccc}
\hline Instrumento & Año & Autores & Perspectiva & Foco & Unidad de análisis \\
\hline TSA & 1999 & Grawe et al. & 0 & $\mathrm{P}$ & 10 minutos \\
\hline CMP & NP & Gassmann & 0 & Py $T$ & 1 minuto \\
\hline ROMA-T/P & $2008 a$ & Flückiger \& Grosse Holtforth & 0 & Py $T$ & 1 minuto \\
\hline BPSR- T/P & 2010 & Flückiger et al. & Py T & Py $T$ & Sesión \\
\hline SACiP & 2013 & Mander et al. & Py T & Py $T$ & Sesión \\
\hline ITPQ & 2015 & Mander et al. & Py T & Py $T$ & Sesión \\
\hline FEPiG & 2016 & Vogel, Blanck, Bents \& Mander & Py T & Py T & Sesión \\
\hline
\end{tabular}

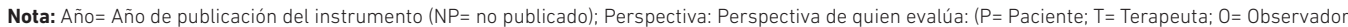
externo); Foco: Sujeto en quien se observa el fenómeno: ( $P$ = Paciente; $T$ = Terapeuta); Unidad de Análisis: duración temporal de episodios que observa.

Los instrumentos Therapy Spectrum Analysis (TSA; Grawe, Regli, Smith, \& Dick, 1999), Consistency Theory Micro-Process Analyses (CMP; Gassmann, 2002) y Ressourcenorientierte Mikroprozessanalyse (ROMA-T/P; Flückiger \& Grosse Holtforth, 2008a) fueron desarrollados para evaluar la conducta explícita de pacientes y terapeutas desde la perspectiva de observadores externos al proceso terapéutico.

El instrumento TSA consiste en un total de 72 escalas que evalúan distintas dimensiones de la experiencia explicitada por el paciente durante la sesión. Las escalas relacionadas con Activación de Recursos incluyen: Calidad del vínculo terapéutico; Recursos relevantes para la participación del paciente en terapia (responsividad a las intervenciones del terapeuta y motivación para realizar contribuciones propias); y Fortalezas generales del paciente, independientes de la terapia (ej: objetivos, habilidades, relaciones de apoyo, etc.). La evaluación de dichas escalas considera, tanto la cantidad de tiempo que estos aspectos son explícitamente discutidos como el grado en que estos son activados en sesión (ej: grado en que el paciente es capaz de explicitar sentimientos de autoeficacia; o motivación respecto de objetivos, etc.), pudiendo tomar cualquier valor entre 0 y 100.

El instrumento CMP corresponde a una tesis no publicada de su autor. A partir de su descripción en el artículo de Gassmann y Grawe (2006), es posible conocer que este instrumento evalúa cuatro dimensiones de la interacción entre paciente y terapeuta, a saber: Contenido de la interacción; Grado de Activación de Recursos; Grado de Activación de Problemas, y; Resultado inmediato de la interacción. En dicho artículo no se describe la operacionalización o criterios de codificación para estas dimensiones. 
El instrumento ROMA evalúa exclusivamente el mecanismo Activación de Recursos, tanto en la actividad del terapeuta como la del paciente, respecto a tres áreas: Competencias (fortalezas personales e interpersonales); Objetivos personales (objetivos, motivaciones e intentos de solución), y; Reinterpretación positiva de problemas (ej: normalización; reencuadre, metáforas personales, etc.). Estas áreas son evaluadas, tanto en un nivel dicotómico (indicador específico observado/indicador específico no observado) como respecto a su cualidad en una escala tipo Likert de 5 puntos, en un rango desde -2 hasta 2 (desde desatender los recursos del paciente, hasta un énfasis explícito de la contribución del paciente en un contexto específico).

Por otro lado, los instrumentos Bern Post Session Report (BPSR-P/T; Fluckiger et al., 2010; Regli \& Grawe, 2000), Multiperspective Assessment of General Change Mechanisms in Psychotherapy (SACiP; Mander et al., 2013), Individual Therapy Process Questionnaire (ITPQ; Mander et al., 2015) y Form for the Evaluation of Therapeutic Processes in Group Therapy (FEPiG; Vogel, Blanck, Bents, \& Mander, 2016), fueron diseñados como cuestionarios de auto reporte para pacientes y terapeutas que deben ser contestados posterior a la sesión.

El instrumento BPSR evalúa distintos constructos relacionados con el proceso terapéutico. Los ítems correspondientes al mecanismo Activación de Recursos son evaluados en una escala tipo Likert de 5 puntos en la versión para terapeutas y en una escala de 7 puntos en la versión para pacientes. Algunos ejemplos de las versiones para terapeuta y paciente respectivamente son: "Hoy traté de hacer un uso específico de los recursos del paciente"; "Hoy día, el terapeuta me ayudó a sentir dónde están mis fortalezas" (Flückiger, Regli, Zwahlen, Hostettler, \& Caspar, 2010, p 74. Traducción propia).

El instrumento SACiP incorpora y modifica ítems de la escala BPSR, y agrega otros para evaluar la Alianza Terapéutica, basado en el instrumento Working Alliance Inventory (Horvath \& Greenberg, 1986) en su forma abreviada y adaptada al alemán (WAI-SR; Munder et al., 2010). Las modificaciones realizadas a los ítems del instrumento BPSR tienen por fin homologar las versiones para terapeutas y pacientes en cuanto a su cantidad, contenido, redacción y rango de las escalas de evaluación (Mander et al., 2013). En el instrumento SACiP, a diferencia del BPSR, todos los ítems son evaluados en escalas tipo Likert de 5 puntos. Los ítems resultantes para evaluar Activación de Recursos, tanto para terapeutas como pacientes son tres: "En la sesión de hoy, el paciente (yo), pudo (pude) sentir dónde están sus (mis) fortalezas; A través de la sesión de hoy, el paciente (yo) pudo (pude) mejorar su (mi) autoimagen; Hoy día, yo, (el terapeuta) usé (usó) intencionalmente las habilidades del paciente (mis habilidades) a favor de la terapia" (Mander et al., 2013, p 108. Traducción propia. Las variaciones de la forma para pacientes se muestran entre paréntesis).

El instrumento ITPQ incorpora ítems de los instrumentos SACiP y Scale of the Therapeutic Alliance-Revised (STA-R; Brockmann et al., 2011), además de desarrollar nuevos ítems para evaluar los constructos Temores del paciente e Interferencia del Terapeuta, desarrollados por Hatcher y Shannon (2005). Los ítems para evaluar Activación de Recursos son idénticos al instrumento SACiP.

Finalmente, el instrumento FEPiG, recoge y adapta los ítems del instrumento SACiP al contexto de terapia grupal, e incorpora ítems para evaluar la variable Cohesión Grupal a partir de la escala Therapeutic Factor Inventory (TFI; MacNair-Semands, Ogrodniczuk \& Joyce, 2010). Para evaluar Activación de Recursos el instrumento 
FEPiG utiliza los 3 ítems de la escala SACiP e incorpora 3 adicionales: "Después de la sesión grupal de hoy, tengo más confianza en mí mismo; La reunión del grupo de hoy me dio valor; Las actividades y el contenido de la reunión del grupo de hoy me dieron esperanza" (Vogel et al., 2016, p 174. Traducción propia.).

\section{Relación entre el mecanismo Activación de Recursos y otras variables}

Los artículos seleccionados, en conjunto, ofrecen evidencia respecto de la relación entre el mecanismo de Activación de Recursos y tres aspectos de la psicoterapia, a saber: Resultados terapéuticos $(n=10)$; Variables de proceso $(n=6), y$; Entrenamiento de terapeutas $(n=1)$. Respecto de los resultados terapéuticos, los artículos ofrecen evidencia respecto de resultados finales de tratamiento $(n=10)$ y resultados evaluados en distintos momentos de la terapia $(n=3)$. Respecto de las variables de proceso, los artículos aportan evidencia respecto de la relación de Activación de Recursos con otros mecanismos genéricos descritos por Grawe (1997) ( $n=4)$, en adelante abreviados como MGG; Alianza Terapéutica (Bordin, 1979) ( $n=3)$, y; Etapas de Cambio (Prochaska \& DiClemente, 1983) $(n=2)$.

\section{Activación de Recursos y Resultados terapéuticos}

Entre los diez artículos que aportan evidencia respecto de resultados terapéuticos, ocho de ellos presentan evidencia a favor de la relación entre el mecanismo de Activación de Recursos y resultados terapéuticos, uno presenta evidencia dividida y uno en contra:

\section{Evidencia a Favor}

Gassmann y Grawe (2006) exploran la relación entre los mecanismos Activación de Problemas y Activación de Recursos, así como la relación de dichos mecanismos con los resultados terapéuticos finales. Los autores reportan que, en terapias con bajos resultados terapéuticos, los terapeutas realizaban una cantidad significativamente menor de intervenciones activadoras de recursos, comparado con los terapeutas de terapias con resultados moderados $(\mathrm{N}=30, \mathrm{t}=-2.8, \mathrm{df}=18, \mathrm{p}<.05)$ y resultados altos $(\mathrm{N}=30, \mathrm{t}=-2.9, \mathrm{df}=17, \mathrm{p}<.05)$. No se encontraron diferencias significativas entre los terapeutas de terapias con resultados moderados y altos.

Flückiger et al. (2009) evalúan los efectos de estrategias específicas de Activación de Recursos en los resultados terapéuticos, y reportan una correlación significativa entre la cantidad total de intervenciones activadoras de recursos realizadas por el terapeuta y cambios en la evaluación de la autoestima de los consultantes evaluados pre y post tratamiento $(r=.52, p<.05)$. Adicionalmente, los autores reportan una correlación significativa entre la calidad de las intervenciones activadoras de recursos (desde ignorar completamente los recursos hasta el énfasis explícito y contextualizado) y el logro de objetivos terapéuticos $(r=.39, p<.01)$.

Znoj et al. (2010) exploran mecanismos que podrían favorecer los resultados terapéuticos de una intervención grupal. Los mecanismos evaluados son: Activación de Recursos, Maestría, Clarificación, Activación de Problemas, Alianza Terapéutica, Progreso en la Terapia, Autoeficacia, y Experiencias Correctivas. Todos los mecanismos evaluados, excepto Activación de Problemas, mostraron alguna correlación significativa con resultados en al menos uno de los momentos del proceso terapéutico. Sin embargo, sólo los mecanismos Activación de Recursos y Progreso en la Terapia mostraron correlaciones significativas con resultados en todas las etapas del proceso. Específicamente, respecto al mecanismo de Activación de Recursos, 
Pág 41

Mander et al. (2014) exploran la relación entre los Mecanismos Genéricos descritos por Grawe (MGG) y Alianza Terapéutica con los resultados terapéuticos finales, reportando que tres variables son capaces de predecir resultados terapéuticos, a saber: Activación de Recursos $(F(1,231.98)=$ $14.43, p<.001)$ Vínculo Emocional $(F(1,227.56)=10.90$, $\mathrm{p}<.001)$; y Clarificación F(1, 230.92) $=13.23, p<.001)$. los autores reportan las siguientes correlaciones con resultados terapéuticos: Fase inicial $(r=.42, p<.05)$; Fase media $(r=.48, p<.05)$; Fase final $(r=.45, p<.05)$, y; Terapia completa $(r=.5, p<.01)$.

Stangier, Von Consbruch, Schramm y Heidenreich (2010) comparan el grado en que el trabajo terapéutico se enfoca en el uso de MGG entre dos terapias manualizadas (Terapia Cognitiva y Terapia Interpersonal) para el tratamiento de la Fobia Social, y su relación con los resultados terapéuticos. Desde la perspectiva del terapeuta, los autores reportan un nivel significativamente más alto a favor de la Terapia Cognitiva en cuanto al grado en que la actividad terapéutica se centra en algunos MGG, entre ellos Activación de Recursos $(F(1,60)=11,66, p<.01)$. Sin embargo, esta diferencia no se encuentra desde la perspectiva del paciente. Los autores no encontraron tampoco diferencias significativas entre ambos enfoques terapéuticos, por sí mismos, respecto de los resultados terapéuticos. Por otro lado, entre 24 modelos predictivos realizados, que incluyen cada una de las variables incluidas en el estudio, evaluadas tanto desde la perspectiva del terapeuta como del consultante, así como la interacción entre ellas, sólo se observaron efectos significativos para dos modelos, siendo la variable Activación de Recursos, evaluado desde la perspectiva del terapeuta, el principal predictor de resultados del estudio, independiente del enfoque terapéutico $(B=1.458, S E=.584$, Wald $=6.244, p=.012, \operatorname{Exp}(B)=4.299)$. El segundo modelo con efectos significativos fue para la variable Activación de Problemas $(B=1.130, S E=$ .566, Wald=3.986, $p=.046, \operatorname{Exp}(B)=3.097)$, que sólo se observó en el grupo de Terapia Conductual.

Mander et al. (2013) presentan la construcción y validación del instrumento SACiP, y evalúan su capacidad para predecir resultados terapéuticos. Desde la perspectiva del terapeuta se encuentran correlaciones significativas con resultados terapéuticos para las variables: Vínculo Emocional; Acuerdo en Colaboración (categoría que incluye Acuerdo sobre Metas y Acuerdo sobre Tareas); Maestría, y; Activación de Recursos. Siendo esta última variable la que presenta la correlación más alta $(r=.16, p<.05)$. Desde la perspectiva del paciente Activación de Recursos muestra una correlación significativa positiva $(r=.22, p<.05)$, y ocupa un cuarto lugar luego de Acuerdo de Colaboración $(r=.24, p<.001)$, Clarificación $(r=.23, p<.001)$ y Maestría $(r=.23, p<.001)$.

Mander et al. (2014) exploran la relación entre los MGG y Alianza Terapéutica con los resultados terapéuticos finales, reportando que tres variables son capaces de predecir resultados terapéuticos, a saber: Activación de Recursos $(F(1,231.98)=$ 14.43, $p<.001)$ Vínculo Emocional $(F(1,227.56)=10.90, p<.001)$; y Clarificación $F(1$, 230.92)=13.23, $p<.001)$.

Mander et al. (2015a) estudian la relación entre los distintos MGG y Esquemas Maladaptativos Tempranos (EMT) definidos por Young (1990), así como la relación entre dichas variables y resultados terapéuticos. Los MGG fueron evaluados desde las perspectivas de pacientes y de terapeutas. Los datos del estudio muestran que sólo la evaluación desde la perspectiva del paciente mostró correlaciones significativas entre algunos MGG y resultados globales del tratamiento. Específicamente, Activación de Recursos mostró una correlación con reducción de sintomatología general $(r=.23, p<.05)$ y estrés percibido $(r=.33, p<.01)$, pero no con problemas interpersonales, que fue la tercera variable utilizada para evaluar resultados terapéuticos. Ninguno de los MGG mostró correlaciones con más de dos de las tres variables utilizadas para evaluar resultados terapéuticos. Adicionalmente, los autores realizaron análisis de correlación entre cada uno de los MGG y los EMT, los cuales arrojaron correlaciones 
Mander et al. (2015b) presentan el desarrollo del instrumento ITPQ. A través del análisis factorial exploratorio, los autores observan que los ítems para evaluar Activación de Recursos cargan en el mismo factor que los ítems que evalúan Maestría por lo que consideran ambos mecanismos como uno solo, al cual denominan "Recursos y Maestría". negativas significativas entre Activación de Recursos, Vínculo y Maestría y los ETM Desconexión y Rechazo; Deterioro de Autonomía y Desempeño, y; Deterioro de Límites. Todas las correlaciones estuvieron en el rango $-.42 \leq r \leq-.29$, y se observaron sólo desde la perspectiva de pacientes. Adicionalmente, a través de distintos modelos de regresión para cada uno de los mecanismos del estudio, se observó un efecto predictivo significativo bidireccional de Activación de Recursos sobre (la disminución de) Deterioro de límites ( $\beta=-.18$ and $p=.036$ ), y (la disminución de) Deterioro de límites sobre Activación de Recursos $(\beta=-.21$ and $p=.044)$. Ningún otro mecanismo mostró efectos significativos en los modelos de regresión realizados.

Mander et al. (2015b) presentan el desarrollo del instrumento ITPQ. A través del análisis factorial exploratorio, los autores observan que los ítems para evaluar Activación de Recursos cargan en el mismo factor que los ítems que evalúan Maestría por lo que consideran ambos mecanismos como uno solo, al cual denominan "Recursos y Maestría". Posteriormente, a través de una serie de modelos de efectos mixtos realizados (uno por cada mecanismo), reportan que cada uno de los mecanismos muestra una relación significativa con los resultados terapéuticos finales. Desde la perspectiva del paciente, el mecanismo Recursos y Maestría, en particular, fue capaz de predecir resultados terapéuticos $(F(1,1522.86)=12.71, p<0.001)$. Adicionalmente, desde esta misma perspectiva se detallan correlaciones significativas entre el mecanismo Recursos y Maestría y severidad de sintomatología $(0.14, p<.05)$, estrés percibido $(0.22, p<.01)$ y experiencia durante la internación $(0.41, p<.01)$. Desde la perspectiva del terapeuta se observó una correlación significativa con estas mismas variables de resultados $(0.15, p<.01 ; 0.23, p<.01, y ; 0.16, p<.05$, respectivamente). No se encontraron correlaciones significativas entre el mecanismo Recursos y Maestría y la variable de resultados terapéuticos Problemas Interpersonales, desde ninguna de las perspectivas.

\section{Evidencia dividida}

Flückiger, Zinbarg, Znoj y Ackert (2014) exploran la temporalidad ("timing") y frecuencia de expresión de las variables Activación de Recursos y Expresión de Emocionalidad Negativa, y la relación entre la aparición de cada una de estas variables durante la fase inicial del proceso terapéutico con los resultados terapéuticos finales. Los autores realizan un análisis de predicción múltiple para las variables Activación de Recursos y Expresión de Emocionalidad Negativa, reportando que ambos juntos logran predecir el $62 \%$ de la varianza del resultado final de la terapia $(F(4,19)=6.1 ; p<.01)$. Ahora bien, respecto de la variable Activación de Recursos, por sí sola, exploran la correlación entre sub-temas, evaluados desde la perspectiva de los pacientes, con resultados terapéuticos; y reportan que el sub-tema "Competencias" mostró una correlación significativa ( $r=.56 \mathrm{p}<.01$ ), y los otros dos sub-temas, a saber, Objetivos Personales y Reinterpretaciones Positivas, mostraron correlaciones negativas con resultados terapéuticos ( $r=$ $-.68 p<.01)$ y $(r=-.67 p<.01)$, respectivamente. Con relación a los resultados negativos, Flückiger et al. (2014) señalan que las características de los participantes (pacientes con diagnóstico de Trastorno de Ansiedad Generalizada, con alto nivel de sintomatología ansiosa) y la revisión de fichas clínicas de los casos, permiten suponer que los contenidos de las tematizaciones observadas de Objetivos Personales pudieran haber estado relacionados con expresión de preocupaciones, más que dar cuenta de la capacidad de autorreflexión de los pacientes; y, que las Reinterpretaciones Positivas observadas pudieran ser parte de mecanismos de regulación de emociones negativas, más que una autoafirmación de sus capacidades. 
Pág 43

Smith y Grawe (2003) exploran la relación entre 23 distintas variables de proceso y la experiencia de Productividad de la sesión, la cual es medida a través de la evaluación del paciente respecto de los mecanismos Maestría, Clarificación y Autoeficacia. Entre las variables evaluadas, el mejor predictor de Productividad resultó ser Activación de Recursos; específicamente, el ajuste entre las intervenciones del terapeuta y las habilidades y recursos del consultante, denominado como Responsividad.

\section{Evidencia en contra}

Sander, Zobel, Dykierek y Schramm (2013) exploran la relación entre los distintos MGG, Alianza Terapéutica y los resultados globales de la terapia. Los autores realizaron distintos análisis de varianza para cada una de las variables y dos grupos: terapias con y sin resultados terapéuticos. Los autores reportan resultados significativos para sólo dos de las variables, únicamente cuando estas son evaluadas desde la perspectiva del paciente, a saber: Maestría $(F(1,28)=7.38,<.05)$ y Clarificación $(F(1,28)=5.98,<.05)$. No se encontraron resultados significativos para Activación de Recursos ni otra variable sobre resultados terapéuticos desde ninguna de las dos perspectivas.

\section{Activación de Recursos y Variables de proceso}

Entre los artículos seleccionados, siete de ellos aportan evidencia a favor de la relación entre el mecanismo Activación de Recursos y tres variables de proceso distintas, a saber: Etapas de Cambio y otros MGG provenientes del marco conceptual de Grawe (1997):

Activación de Recursos y otros MGG

Smith y Grawe (2003) exploran la relación entre 23 distintas variables de proceso y la experiencia de Productividad de la sesión, la cual es medida a través de la evaluación del paciente respecto de los mecanismos Maestría, Clarificación y Autoeficacia. Entre las variables evaluadas, el mejor predictor de Productividad resultó ser Activación de Recursos; específicamente, el ajuste entre las intervenciones del terapeuta y las habilidades y recursos del consultante, denominado como Responsividad. Cuando este ajuste ocurre, la probabilidad de que el paciente experimente la sesión como productiva es de $61 \%$. En cambio, cuando el paciente siente las intervenciones del terapeuta como lejanas a sus capacidades, la probabilidad de la experiencia de Productividad de la sesión desciende a $21 \%$. La siguiente variable que contribuye con mayor peso en esta experiencia, siempre que coexista con un alto nivel de Responsividad, es la manera de abordar los problemas: Mientras mayor sea el énfasis hacia el cambio, mayor es la probabilidad de que el paciente lo perciba como productivo, llegando a 85\%. En cambio, sí el énfasis de los problemas está puesto en estados, la probabilidad de experiencia de productividad se reduce a $41 \%$. Ahora bien, ante un alta Responsividad y énfasis de los problemas puesto en cambios, los autores reportan que el patrón más productivo ocurre cuando los problemas no son altamente activados emocionalmente ( $98 \%$ de probabilidad de productividad). En contraste, el patrón de menor probabilidad de Productividad corresponde a: baja Responsividad, bajo reconocimiento de los recursos del paciente por parte del terapeuta y mala calidad de vínculo terapéutico ( $2 \%$ de probabilidad de experiencia de Productividad de la sesión).

Smith y Grawe (2005) investigan patrones de combinación de distintos mecanismos de cambio (Activación de Recursos; Vínculo Terapéutico; Involucración del Terapeuta; Discusión de Problemas; Activación de Problemas; y Contribución del Paciente en la discusión del Problema), y señalan que, durante la primera mitad de la terapia, se observan múltiples patrones relacionados con una alta experiencia de Productividad por parte del paciente, pudiendo estar presentes o faltar distintos mecanismos, excepto Activación de Recursos; cuando este último falta, el paciente suele percibir la sesión como poco productiva. El patrón con mayor frecuencia de baja Productividad (16\% de los casos) incluye: buena Calidad del Vínculo; alto nivel de Contribución del terapeuta y paciente en la discusión de problemas; alto nivel de Activación de Problemas, y; bajo nivel de Activación de Recursos. A medida que la terapia alcanza su fase media 
Al igual que Smith y Grawe (2003; 2005), para evaluar resultados de la sesión, utilizan la evaluación del paciente respecto de los mecanismos Clarificación y Maestría. Los autores reportan que en sesiones evaluadas como "exitosas" los terapeutas realizan intervenciones de Activación de Recursos con una frecuencia, en todo momento, por sobre la media de la muestra, con un incremento considerable al inicio y final de estas sesiones. el patrón con mayor frecuencia de experiencia de Productividad de la sesión implica que ocurran los siete procesos evaluados en un nivel alto ( $15 \%$ de los casos). Y, de la mitad de la duración de la terapia en adelante, los patrones en que se observa mayor experiencia de productividad incluyen: una disminución del foco en los mecanismos de Tematización de Problemas y Activación de Problemas, manteniendo alto el nivel de Activación de Recursos (14\% de los casos).

Gassmann y Grawe (2006) realizan distintos análisis de micro proceso respecto de las conductas de Activación de Recursos en terapeutas y consultantes en sesiones "exitosas" y "no exitosas". Al igual que Smith y Grawe (2003; 2005), para evaluar resultados de la sesión, utilizan la evaluación del paciente respecto de los mecanismos Clarificación y Maestría. Los autores reportan que en sesiones evaluadas como "exitosas" los terapeutas realizan intervenciones de Activación de Recursos con una frecuencia, en todo momento, por sobre la media de la muestra, con un incremento considerable al inicio y final de estas sesiones. En cambio, en las sesiones "no exitosas", se observa que los terapeutas rara vez realizan alguna intervención de Activación de Recursos antes del último cuarto de la sesión. Adicionalmente, en sesiones "no exitosas" los autores refieren la existencia de una correlación cruzada negativa significativa, a lo largo de la sesión, entre la conducta de Activación de Recursos de terapeuta y paciente. Respecto a estos análisis solo se reportan datos expresados gráficamente.

Flückiger et al. (2009) analizan el efecto aislado de las intervenciones de Activación de Recursos, desagrupadas en tres "clusters", según el foco temático de la intervención, a saber: a) Competencias personales e interpersonales; b) Objetivos personales, y; c) Resignificaciones positivas. Específicamente, las intervenciones enfocadas en las Competencias de los consultantes mostraron correlaciones significativas con Alianza Terapéutica $(r=.37, p<.01)$, Activación de Problemas, $(r=.48, p<.01)$ y Maestría $(r=.44, p<.01)$; las intervenciones enfocadas en Objetivos mostraron una correlación significativa con Activación de Problemas $(r=.36, p<.01)$, Maestría $(r=.35, p<.01)$ y Clarificación ( $r=.45, p<.05)$, y; las enfocadas en Reinterpretaciones Positivas, mostraron un correlación inversa significativa con Activación de Problemas $(r=-.35, p<.01)$. A su vez, la suma total de intervenciones activadoras de recursos mostró una correlación significativa con los mecanismos de Activación de Problemas ( $r=.36, p<.01)$, Maestría $(r=.45, p<.01)$ y Clarificación $(r=.41, p<.01)$.

\section{Activación de Recursos y Etapas de Cambio}

Mander et al. (2014) exploran la relación entre las Etapas de Cambio (EC) descritas por Prochaska y DiClemente (1983), y los distintos MGG. La hipótesis central del estudio es que aquellos MGG que definen como Experienciales (Vínculo Terapéutico; Activación de Problemas; y Clarificación) debieran ser especialmente importantes en las EC tempranas (Precontemplación y Contemplación), mientras que aquellos que definen como Conductuales (Activación de Recursos, Maestría y Acuerdo de Colaboración), serían más importantes en etapas avanzadas (Acción y Mantenimiento). Al respecto, los autores reportan que la EC Contemplación, en una fase inicial del proceso terapéutico, predice la aparición de mecanismos Conductuales hacia el final del tratamiento: $(b=.15, p<.05)$. Y que, tanto los mecanismos Experienciales como los Conductuales, durante la fase inicial del proceso, predicen la EC Acción hacia el final del tratamiento: $(b=.13, p<.05)$ y $(b=.16, p<.05)$ respectivamente. Para el mecanismo de Activación de Recursos, específicamente, se encuentran las siguientes correlaciones significativas: Durante la fase inicial 
del tratamiento: Contemplación $(r=.15,<.05)$, Acción $(r=.27,<.01)$, y Mantenimiento $(r=.16,<.05)$; durante la fase media del tratamiento: Acción $(r=.37,<.01)$, y, para la fase final del tratamiento: Acción $(r=.49,<.01)$.

Schaller et al. (2018) exploran la relación entre MGG y las EC, aplicado al contexto de intervención de psicoterapia grupal de corta duración. Las variables de dicho estudio fueron evaluadas al inicio (t1: segunda sesión) y final de la terapia (t2: séptima sesión). Respecto a los resultados del estudio, los autores reportan que los mecanismos Experienciales, al inicio de la terapia (t1), predicen un alto puntaje para Contemplación hacia el final de la terapia (t2): $(\beta=.20 ; p<.01)$. A su vez, altos niveles de mecanismos Conductuales al inicio de la terapia (t1), predicen un alto puntaje para Acción hacia el final de la terapia (t2): ( $\beta=.17 ; p<.05)$. Para el mecanismo de Activación de Recursos, medido de forma aislada, cuando este es evaluado al inicio de la terapia (t1), correlaciona significativamente con Contemplación (t1) ( $r=.18 ; p<.05$ ), Acción (t1) ( $r=.49 ; p<.001)$ y Mantenimiento (t1) ( $r=.21 ; p<.05)$, al inicio de la terapia, así como con Contemplación $(r=.17 ; p<.05)$ y Acción $(r=.41 ; p<.001)$ hacia el final de la terapia (t2).

\section{Activación de Recursos y entrenamiento de terapeutas}

Flückiger y Grosse Holtforth (2008b) evalúan los efectos de una intervención/entrenamiento para terapeutas, cuyo objetivo es favorecer que estos aumenten el grado en que realizan intervenciones activadoras de recursos a lo largo del tratamiento. Para esto diseñaron un estudio no aleatorizado con grupo control, en el que los terapeutas del grupo de intervención: a) Previo a la primera sesión, discutían con un estudiante de magister entrenado en Activación de Recursos acerca de una evaluación realizada por el paciente sobre sus propios recursos; y b) Inmediatamente posterior a cada una de las primeras cinco sesiones, sostenían una reunión de cinco minutos, con el mismo estudiante de magister, acerca de cómo habían implementado intervenciones orientadas hacia la Activación de Recursos y qué nuevas intervenciones podría realizar en la próxima sesión. Los terapeutas del grupo de intervención, a través de la subescala Activación de Recursos del instrumento BPSR-T, evaluaron más alto que el grupo control respecto a haber trabajado con los recursos de los consultantes, durante las primeras cinco sesiones. La mayor diferencia entre ambos grupos se observó en la sesión $3\left(\beta_{01}(E X P)=.57, t=3.8, p<.001, d=.63\right)$. Esta diferencia tendía a desaparecer luego de la quinta sesión, una vez que ya no se sostenían las reuniones entre el terapeuta y el estudiante. No se realizó una evaluación de esta variable desde la perspectiva de los pacientes. Desde la perspectiva de observadores externos, ciegos a las condiciones de tratamiento, se encontraron diferencias significativas, a favor del grupo de intervención, respecto de la actividad de los pacientes relacionada con Activación de Recursos ( $\beta_{01}$ (EXP)5.13, t52.94, p<.001); sin embargo, cuando evaluaron la conducta de los terapeutas, no observaron diferencias significativas entre los grupos. Adicionalmente, los autores evalúan desde la perspectiva de terapeutas y pacientes, distintos efectos de corto y largo plazo de Activación de Recursos sobre Maestría y Alianza Terapéutica, encontrando diferencias significativas entre los grupos, a favor del grupo de intervención, sólo cuando fue evaluado desde la perspectiva de los terapeutas. Por otro lado, se evaluó la diferencia entre grupos respecto de los resultados terapéuticos finales (evaluado por los pacientes), encontrando diferencias significativas a favor del grupo de intervención respecto de la variable Severidad de Sintomatología $(F(1,38)=52.84, p<.05)$, no así respecto de Emocionalidad ni de Problemas Interpersonales, que fueron las otras dos variables de resultados utilizadas. Cabe señalar que los pacientes eran ciegos respecto a su pertenencia a los grupos de intervención y control. 
Las investigaciones desarrolladas por Gassmann y Grawe (2006), y Smith y Grawe (2003; 2005) permiten suponer que el mecanismo de Activación de Recursos podría desempeñar un papel de condición mínima, pero no suficiente, para la puesta en marcha y progreso del proceso terapéutico. En este sentido, las intervenciones orientadas hacia la Activación de Recursos, por sí mismas, no asegurarían el cambio e, incluso, podrían ser contraproducentes en determinadas circunstancias (Flückiger et al., 2014; Schaller et al., 2018; Smith \& Grawe, 2005).

\section{Discusión}

A partir de los estudios seleccionados en la presente revisión, se encuentra evidencia mayoritariamente favorable respecto a la relación entre el mecanismo de Activación de Recursos y los resultados terapéuticos. La evidencia en contra o dividida acerca de esta relación, observada en dos de los artículos (Flückiger et al., 2014; Sander et al., 2013), es posible pensar que obedece, al menos en parte, a características metodológicas de los estudios (tamaño muestral; análisis estadísticos realizados; sensibilidad y especificidad de los instrumentos utilizados, etc.). Respecto del estudio reportado por Sander et al. (2013), distintos aspectos de la metodología utilizada, incluido el diseño metodológico y el tamaño muestral, pudieran haber tenido un impacto en los hallazgos, no sólo respecto de Activación de Recursos, sino también de otras variables del estudio como, por ejemplo, Alianza Terapéutica, para la cual no se observó efectos significativos, a pesar de la amplia evidencia empírica respecto de la relación de esta variable con resultados terapéuticos (Flückiger, Del Re, Wampold, \& Horvath, 2018; Norcross \& Wampold, 2011). Y, en el estudio reportado por Flúckiger et al. (2014), por otro lado, el mecanismo Activación de Recursos se evaluó a través de ROMA, que mide presencia de temáticas relacionadas con este mecanismo y el grado de contribución de terapeuta y paciente a dicha tematización, pero no considera el contenido específico de estas tematizaciones, por lo que la explicación de los autores respecto de los resultados negativos observados es plausible. Por ejemplo, tanto terapeuta como paciente pudieran tematizar activa y colaborativamente acerca de objetivos personales del paciente, sin embargo, el énfasis de esta tematización pudiera estar puesto en temores o experiencias previas de fracaso y, aun así, el instrumento podría identificar dicho episodio como Activación de Recursos.

Ahora bien, respecto de distintos modos posibles de uso del mecanismo Activación de Recursos, cuándo utilizarlo o cómo entrenarlo para favorecer un mayor provecho terapéutico, la evidencia es bastante más limitada. Las investigaciones desarrolladas por Gassmann y Grawe (2006), y Smith y Grawe (2003; 2005) permiten suponer que el mecanismo de Activación de Recursos podría desempeñar un papel de condición mínima, pero no suficiente, para la puesta en marcha y progreso del proceso terapéutico. En este sentido, las intervenciones orientadas hacia la Activación de Recursos, por sí mismas, no asegurarían el cambio e, incluso, podrían ser contraproducentes en determinadas circunstancias (Flückiger et al., 2014; Schaller et al., 2018; Smith \& Grawe, 2005). De este modo, es necesario identificar cuándo, frente a quién y qué aspecto del mecanismo de Activación de Recursos poner en acción o enfatizar, en cada momento. Para comprender mejor estos aspectos se requiere todavía de mayor desarrollo de investigaciones de micro proceso. A modo de referencia, los estudios de Flückiger, Zinbarg, Znoj y Ackert (2014), y Smith y Grawe (2003; 2005), resultan interesantes respecto del uso de metodologías sofisticadas para esclarecer dichos aspectos, aunque debido a su complejidad y tamaño muestral requieren una inversión de recursos considerables para ser replicados. Por otro lado, el estudio de Gassmann y Grawe (2006) resulta interesante respecto al nivel de detalle específico y a su contribución al entendimiento de micro procesos, a partir de números muestrales relativamente pequeños y análisis estadísticos sencillos, siendo una alternativa comparativamente más plausible de replicar en contextos naturalistas y con una menor inversión de recursos. 
Pág 47

Es posible afirmar que, si bien la evidencia de los estudios es mayoritaria a favor de la relación entre Activación de Recursos y resultados terapéuticos, todavía es poco lo que se sabe sobre el modo de explotar el máximo potencial de este mecanismo para promover el cambio psicoterapéutico.

\section{Limitaciones}

Las principales limitaciones del presente estudio se refieren a la heterogeneidad de diseños metodológicos, tipos de análisis estadísticos, tamaño y características diagnósticas de las muestras, y de instrumentos para evaluar Activación de Recursos y variables secundarias de los estudios incluidos. Esto, sumado a la homogeneidad respecto a la comunidad de autores y contexto geográfico-cultural de desarrollo de los estudios, sugieren interpretar con cautela la integración y generalización de la evidencia a la espera de más estudios en distintos contextos. Adicionalmente, debido a limitaciones idiomáticas de los investigadores, se excluyeron artículos que reportaban resultados de estudios empíricos sobre el tema, en alemán, los cuales pudieran haber contribuido a esclarecer, robustecer o matizar algunos de los resultados discutidos en el presente artículo.

\section{Conclusiones}

Finalmente, es posible afirmar que, si bien la evidencia de los estudios es mayoritaria a favor de la relación entre Activación de Recursos y resultados terapéuticos, todavía es poco lo que se sabe sobre el modo de explotar el máximo potencial de este mecanismo para promover el cambio psicoterapéutico. Al mismo tiempo, los estudios de proceso y proceso-resultado sobre Activación de Recursos ofrecen datos promisorios, pero aún exploratorios. Los esfuerzos realizados en los 17 años transcurridos desde el primer artículo seleccionado en la presente revisión hasta la fecha han aportado aspectos prometedores respecto a la comprensión de la contribución del mecanismo Activación de Recursos al cambio en psicoterapia, pero esta comprensión todavía está lejos de agotarse. En un modo figurativo, podría decirse que la investigación sobre Activación de Recursos, si bien ha crecido bastante, no ha cumplido aún su mayoría de edad y se beneficiaría bastante de salir a recorrer el mundo.

\section{Referencias}

Brockmann, J., Kirsch, H., Hatcher, R., Andreas, S., Benz, S., \& Sammet, I. (2011). Dimensions of the Therapeutic Alliance from Patients' View - Development of the "Skala Therapeutische Allianz-Revised STA-R". Psychotherapie Psychosomatik Medizinische Psychologie, 61(5), 208-215. https://doi.org/10.1055/s-0030-1263142

Castronovo, M., Hirsch, H., \& Hirsch, P. (s.f.). Guía y esquema gráfico para el uso de los recursos del consultante en terapia estratégica. Recuperado de http://www. enciclopediasaludmental.org.ar/trabajo.php?idt=14\&idtt=39

Eysenck, H. J. (1994). Personality: Biological foundations. In P. A. Vernon (Ed.), The neuropsychology of individual differences. London: Academic Press. https://doi. org/10.1016/B978-0-12-718670-2.50011-6.

Flückiger, C., Caspar, F., Grosse Holtforth, M., \& Willutzki, U. (2009). Working with patients' strengths: A microprocess approach. Psychotherapy Research, 19(2), 213223. https://doi.org/10.1080/10503300902755300

Flückiger, C., Del Re, A. C., Wampold, B. E., \& Horvath, A. O. (2018). The alliance in adult psychotherapy: A meta-analytic synthesis. Psychotherapy, 55(4), 316-340. https://doi.org/10.1037/pst0000172

Flückiger, C., \& Grosse Holtforth, M. (2008a). Ressourcenorientierte Mikroprozess Analyse (ROMA) - Ressourcendiagnostik und Ressourcenaktivierung im Therapieprozess. (Resource-Oriented Microproces Analysis - ROMA.) [Special issue]. Zeitschrift für Klinische Diagnostik und Evaluation, 1, 171-185. 
Flückiger, C., \& Grosse Holtforth, M. (2008b). Focusing the therapist's attention on the patient's strengths: A preliminary study to foster a mechanism of change in outpatient psychotherapy. Journal of Clinical Psychology, 64, 876-890. https://doi. org/10.1002/jclp.20493

Flückiger, C., Regli, D., Zwahlen, D., Hostettler, S., \& Caspar, F. (2010). Der Berner Patienten- und Therapeutenstundenbogen 2000: Ein Instrument zur Erfassung von Therapieprozessen [The Bern Post Session Report for Patients and for Therapists 2000: An instrument to measure therapy processes]. Zeitschrift für Klinische Psychologie und Psychotherapie:Forschung und Praxis, 39(2), 71-79. https://doi.org/10.1026/1616-3443/a000015

Flückiger, C., Zinbarg, R., Znoj, H., \& Ackert, M. (2014). Resource activation in generalized anxiety: An observer-based microprocess analysis of patients' in-session outcomes. Psychotherapy, 51(4), 535-545. https://doi.org/10.1037/a0034119

Gelso, C. J., \& Woodhouse, S. (2003). Toward a positive psychotherapy: Focus on human strength. En W. B. Walsh (Ed.), Counseling psychology and optimal human functioning (pp 171-198). NY: Erlbaum.

Gassmann, D., \& Grawe, K. (2006). General change Mechanisms: The relation between problem activation and resource activation in successful and unsuccessful therapeutic interactions. Clinical Psychology and Psychotherapy, 13, 1-11. https://doi. org/10.1002/cpp.442

Grawe, K. (1997). Research-informed psychotherapy. Psychotherapy Research, 7, 1-19. https://doi.org/10.1080/10503309712331331843

Grawe K., Donati R., \& Bernauer F. (1994). Psychotherapie im Wandel: von derKonfession zur Profession [The change of psychotherapy. From confession to profession]. Göttingen: Hogrefe.

Grawe, K., Regli, D., Smith, E., \& Dick, A. (1999). Wirkfaktorenanalyse-ein Spektroskop für die Psychotherapie. Verhaltenstherapie und psychosoziale Praxis, 31, 201-225.

Harbin, J. M., Gelso, C. J., \& Rojas, A. E. P. (2014). Therapist work with client strengths: Development and validation of a measure. The Counseling Psychologist, 42(3), 345-373. https://doi.org/10.1177/0011000012470570.

Hatcher, R. L., \& Shannon, P. A. (2005). The patient's collaborative alliance in therapy. unpublished manuscript.

Hirsch, H., Castronovo, M., \& Santangelo, L. (2016). ¿Cuáles son los recursos externos al consultante que el terapeuta puede examinar? De Familias y Terapias, 41, 21-30.

Horvath, A. O., \& Greenberg, L. (1986). The development of the Working Alliance Inventory. En L. S. Greenberg y W. M. Pisnsof, (Eds.), The Psychotherapeutic Process: A Research Handbook (pp. 529-556). Nueva York: Guilford.

Krause, M., \& Altimir, C. (2016). Introduction: Current developments in psychotherapy process research. Estúdios de Psicologia, 37(2-3), 201-225. https://doi.org/10.10 80/02109395.2016.1227574

Lambert, M. J., \& Ogles, B. M. (2004). The efficacy and effectiveness of psychotherapy. In M. J. Lambert (Ed.), Bergin and Garfield's handbook of psychotherapy and behavior change (5th ed.). New York: Wiley.

Lipsey, M. W., \& Wilson, D. B. (2001). Practical meta-analysis. Thousand Oaks: Sage.

Lopez, S. (2008). Positive psychology: Exploring the best in people. Westport, CT: Praeger Publications.

MacNair-Semands, R. R., Ogrodniczuk, J. S., \& Joyce, A. S. (2010). Structure and initial validation of a short form of the Therapeutic Factors Inventory. International Journal of Group Psychotherapy, 60(2), 245-281. https://doi.org/10.1521/ ijgp.2010.60.2.245 
Mander, J., Wittorf, A., Schlarb, A., Hautzinger, M., Zipfel, S., \& Sammet, I. (2013). Change mechanisms in psychotherapy: Multiperspective assessment and relation to outcome. Psychotherapy Research, 23(1), 105-116. https://doi.org/10.108 $\underline{0} / 10503307.2012 .744111$

Mander, J., Wittorf, A., Klingberg, S., Teufel, M., Zipfel, S., \& Sammet, I. (2014). The patient perspective on therapeutic change: The investigation of associations between stages of change and general mechanisms of change in psychotherapy research. Journal of Psychotherapy Integration, 24(2), 122-137. https://doi.org/10.1037/ a0036976

Mander, J., Jacob, G., Götz, L., Sammet, I., Zipfel, S., \& Teufel, M. (2015a). Associations between Grawe's general mechanisms of change and Young's early maladaptive schemas. Psychotherapy Research, 25(2), 249-262. https://doi.org/10.1080/105 03307.2014.889330

Mander, J., Schlarb, A., Teufel, M., Keller, F., Hautzinger, M., Zipfel, S., ... Sammet, I. (2015b). The Individual Therapy Process Questionnaire: Development and Validation of a Revised Measure to Evaluate General Change Mechanisms in Psychotherapy. Clinical Psychology and Psychotherapy, 22, 328-345. https://doi. org/10.1002/cpp.1892

Moher, D., Liberati, A., Tetzlaff, J., Altman, D.G., \& The PRISMA Group. (2009). Preferred Reporting Items for Systematic Reviews and Meta-Analyses: The PRISMA Statement. Open Med, 3(3), 123-130.

Munder, T., Wilmers, F., Leonhart, R., Linster, H.W., \& Barth, J. (2010). Working Alliance Inventory-Short Revised (WAI-SR): Psychometric properties in outpatients and inpatients. Clinical Psychology and Psychotherapy, 17(3), 231-239. https://doi. org/10.1002/cpp.658

Norcross, J. C., \& Wampold, B. E. (2011). Evidence-based therapy relationships: Research conclusions and clinical practices. Psychotherapy, 48(1), 98-102. https:// doi.org/10.1037/a0022161

Regli, D., \& Grawe, K. (2000). Die Berner Stundenbogen Version 2000-ein Forschungsinstrument [The Bern postsession reports-Research instrument. Unpublished manuscript, University of Bern, Bern, Switzerland.

Schaller, G., Blanck, P., Vogel, E., Vonderlin, E., Bents, H., \& Mander, J. (2018). Therapeutic processes in group therapy: Intersections between general change mechanisms and motivational stages of change from patient perspective, European Journal of Psychotherapy \& Counselling, 20(3), 312-336. https://doi.org/10.1 $\underline{080 / 13642537.2018 .1495247 .}$

Scheel, M., Davis, C., \& Henderson, J. (2012). Therapist use of client strengths: a qualitative study of positive processes. The Counseling Psychologist, 20(10) 1-36.

Sander, L., Zobel, I., Dykierek, P., \& Schramm, E. (2013). Common Factors in the treatment of chronic depression. Comparison of 2 psychotherapy methods. Verhaltenstherapie, 22, 228-235. https://doi.org/10.1159/000345644

Seligman, M.E.P., \& Csikszentmihalyi, M. (2000). Positive psychology: An introduction. American Psychologist, 55, 5-14.

Smith, M. L., \& Glass, G. V. (1977). Meta-analysis of psychotherapy outcome studies. American Psychologist, 32, 752-760. https://doi.org/10.1037/0003-066X.32.9.752

Smith, E., \& Grawe, K. (2003). What makes psycho-therapy sessions productive? A new approach to bridging the gap between process research and practice. Clinical Psychology and Psychotherapy, 10, 275-285. https://doi.org/10.1002/cpp.377

Smith, E., \& Grawe, K. (2005). Which therapeutic mechanism Works when? A step towards the formulation of empirically validated guidelines for therapists' session-to-session decisions. Clinical Psychology and Psychotherapy, 12, 112-123. https://doi.org/10.1002/cpp.427 
Stangier, U., Von Consbruch, K., Schramm, E., \& Heidenreich, T. (2010). Common factors of cognitive therapy and interpersonal psychotherapy in the treatment of social phobia. Anxiety, Stress, \& Coping, 23(3), 289-301. https://doi. org/10.1080/10615800903180239

Tarragona, M. (2006). Las Terapias Posmodernas: Una breve Introducción a la Terapia Colaborativa, la Terapia Narrativa y la Terapia Centrada en Soluciones. Psicología Conductual. 14(3) 511-532.

Tarragona, M. (2013). Psicología Positiva y Terapias Constructivas: Una Propuesta Integradora. Terapia psicológica, 31(1), 115-125.

Trösken, A. K., \& Grawe, K. (2003). Das Berner Ressourceninventar-Instrumente zur Erfassung von Patientenressourcen aus der Selbst und Fremdbeurteilsperspektive [The Bern resource inventories-Instruments of assessing patients resources for self and observer report]. In H., Schemmel \& J. Schaller (Eds.), Ressourcen. Ein Hand- und Lesebuch zur therapeutischen Arbeit (pp. 195-217). Tübingen, Germany: dgvt.

Vogel, E., Blanck, P., Bents, H., \& Mander, J. (2016). Wirkfaktoren in der Gruppentherapie: Entwicklung und Validierung eines Fragebogens [Effective factors in group therapy: Development and validation of a questionnaire]. Psychotherapie, Psychosomatik, Medizinische Psychologie, 66(5), 170-179. https://doi. org/10.1055/s-0042-104495

von Consbruch, K., Flückiger, C., Stangier, U., Beutel, M., Herpertz, S., Hoyer, J... Wiltink, J. (2013). WIFA-k: Ein neues Messinstrument zur zeitökonomischen Erfassung allgemeiner Wirkfaktoren nach Grawe. [WIFA-k: A new instrument for a time-efficient assessment of common factors in psychotherapy]. Psychotherapie, Psychosomatik, Medizinische Psychologie, 63, 286-289. https://doi. org/10.1055/s-0032-1331206

Wampold, B., \& Imel, Z. E. (2015). The great psychotherapy debate: The evidence for what makes psychotherapy work (2nd ed.). New York, NY: Routledge.

Young, J. E. (1990). Cognitive therapy for personality disorders: A schema-focused approach. Sarasota, FL: Professional Resource Exchange.

Znoj, H.-J., Messerli-Burgy, N., Tschopp, S., Weber, R., Christen, L., Christen, S., \& Grawe, K. (2010). Psychotherapeutic process of cognitive_behavioral intervention in HIV-infected persons: Results from a controlled, randomized prospective clinical trial. Psychotherapy Research, 20(2), 203-213. https://doi. org/10.1080/10503300903246663 